Trauma Berufskrankh 2008 · 10 [Suppl 2]:241-244

DOI 10.1007/s10039-008-1372-2

Online publiziert: 19. April 2008

(c) Springer Medizin Verlag 2008

G. Gradl · M. Wendt · P. Gierer · M. Beck · T. Mittlmeier Abteilung für Unfall- und Wiederherstellungschirurgie, Chirurgische Klinik und Poliklinik, Universität Rostock

\title{
Intramedulläre Versorgung der distalen Radiusfraktur
}

Die volare winkelstabile Versorgung distaler Radiusfrakturen ist ein Standardverfahren geworden $[5,8,9,10,11]$. Die offene Reposition und interne Fixation erlauben eine direkte Manipulation des Gelenk tragenden Fragments und können auch bei Extensionsfrakturen durch die Wahl winkelstabiler Platten von volar erfolgen [5]. Problematisch jedoch sind $[1,2,11]$ :

- der invasive operative Zugangsweg mit konsekutiver Narbenbildung,

- die Komorbidität der evtl. durchzuführenden Implantatentfernung und

- die meist nicht optimale Rekonstruktion der volaren Kippung in Neutralstellung.

Seit kurzem ist die intramedulläre Versorgung der distalen Radiusfraktur mit einem Hybrid aus Platte und Nagel (Targon ${ }^{\circledR}$ DR, Aesculap, Tuttlingen) möglich. Durch die intramedulläre Lage sollen einerseits ein hohes Maß an Stabilität gewährleistet werden, andererseits auch die Zugangsmorbidität und die sekundären Implantatentfernungen reduziert werden.

Ziel dieser Studie ist es, die intramedulläre Osteosynthese der Radiusfraktur der Standardosteosynthese mit einer volaren winkelstabilen Platte in einem prospektiv randomisierten Setup gegenüber zu stellen.

\section{Methodik}

Im Rahmen einer prospektiv randomisierten Studie wurden 122 Patienten mit distaler Radiusfraktur (AO: $\mathrm{A}_{2-3}, \mathrm{C}_{1-2}$ ) mit einer intramedullären Osteosynthese $\left(\mathrm{n}=66\right.$; Targon $^{\circ} \mathrm{DR}$, Aesculap, Tuttlingen) oder einer volaren, winkelstabilen Platte $(\mathrm{n}=56 ; 2,4-\mathrm{mm}-\mathrm{LCP}$, Clinical House, Bo- chum) operativ versorgt. Das mittlere Alter betrug 63,4 $\pm 12,1$ Jahre.

Das Studienprotokoll erhielt ein positives Votum der lokalen Ethikkommission. Es sah klinische und radiologische Verlaufskontrollen nach 8 Wochen, 6 Monaten und $1 \mathrm{Jahr}$ vor. Hierbei wurden Standardröntgenaufnahmen der betroffenen Hand sowie einmalig der Gegenseite angefertigt. Die Messung der Knochendichte des kontralateralen distalen Radius wurde anhand der quantitativen Computertomographie (pQCT) vorgenommen, wobei angenommen wurde [7]:

- bei einer trabekulären Dichte $>120$ $\mathrm{mg} \mathrm{HA} / \mathrm{cm}^{3}$ eine normale Spongiosadichte,

- bei einem Wert von 120-80 mg HA/ $\mathrm{cm}^{3}$ eine osteopenische Spongiosadichte und

\begin{tabular}{|c|c|c|c|c|c|c|}
\hline Funktion & $\begin{array}{l}\text { Intramedulläre } \\
\text { Osteosynthese } \\
n=66 \\
6 \text { Monate }\end{array}$ & $\begin{array}{l}\text { Volare Platten- } \\
\text { osteosynthese } \\
n=56 \\
6 \text { Monate }\end{array}$ & $\begin{array}{l}\text { Intramedulläre } \\
\text { Osteosynthese } \\
n=54 \\
6 \text { Monate }\end{array}$ & $\begin{array}{l}\text { Volare Platten- } \\
\text { osteosynthese } \\
n=47 \\
6 \text { Monate }\end{array}$ & $\begin{array}{l}\text { Intramedulläre } \\
\text { Osteosynthese } \\
\mathrm{n}=37 \\
1 \mathrm{Jahr}\end{array}$ & $\begin{array}{l}\text { Volare Plattenosteo- } \\
\text { synthese } \\
\mathrm{n}=24 \\
1 \mathrm{Jahr}\end{array}$ \\
\hline Extension [\%] & 81 & $73^{*}$ & 93 & 86 & 97 & 83 \\
\hline Flexion [\%] & 67 & $65^{*}$ & 84 & 79 & 92 & 86 \\
\hline Pronation [\%] & 99 & 96 & 100 & 100 & 100 & 100 \\
\hline Supination [\%] & 89 & 87 & 96 & 99 & 96 & 97 \\
\hline Radialabduktion [\%] & 75 & $67^{*}$ & 93 & 93 & 96 & 95 \\
\hline Ulnarabduktion [\%] & 80 & 74 & 88 & 85 & 91 & 91 \\
\hline Schmerz VAS & 2,1 & $2,8^{*}$ & 1,3 & 1,9 & 0,7 & 0,6 \\
\hline Handkraft [\%] & 45 & 41 & 77 & 80 & 87 & 82 \\
\hline G\&W-Score ${ }^{a}$ & 5,3 & 6,0 & 2,0 & 2,2 & 1,6 & 2,0 \\
\hline CTS $(n)$ & 0 & 0 & 2 & 2 & 2 & 3 \\
\hline Implantatentfernung & 0 & 0 & 2 & 2 & 3 & 8 \\
\hline \multicolumn{7}{|c|}{$\begin{array}{l}\text { Handfunktion } 8 \text { Wochen, } 6 \text { Monate und } 1 \text { Jahr nach Versorgung der distalen Radiusfraktur durch intramedulläre Osteosynthese (Targon }{ }^{\circledast} \text { DR) oder volare Plat- } \\
\text { tenosteosynthese (2,4-mm-Platte) } \\
\text { aGartland-and-Werley-Score: } 0-2 \text { exzellent, 3-8 gut, 9-20 akzeptabel, > } 20 \text { gering } \\
\text { * } p<0,05 \text {, Student-t-Test, Post-hoc-Bonferoni-Korrektur }\end{array}$} \\
\hline
\end{tabular}




\section{Radiusfraktur}
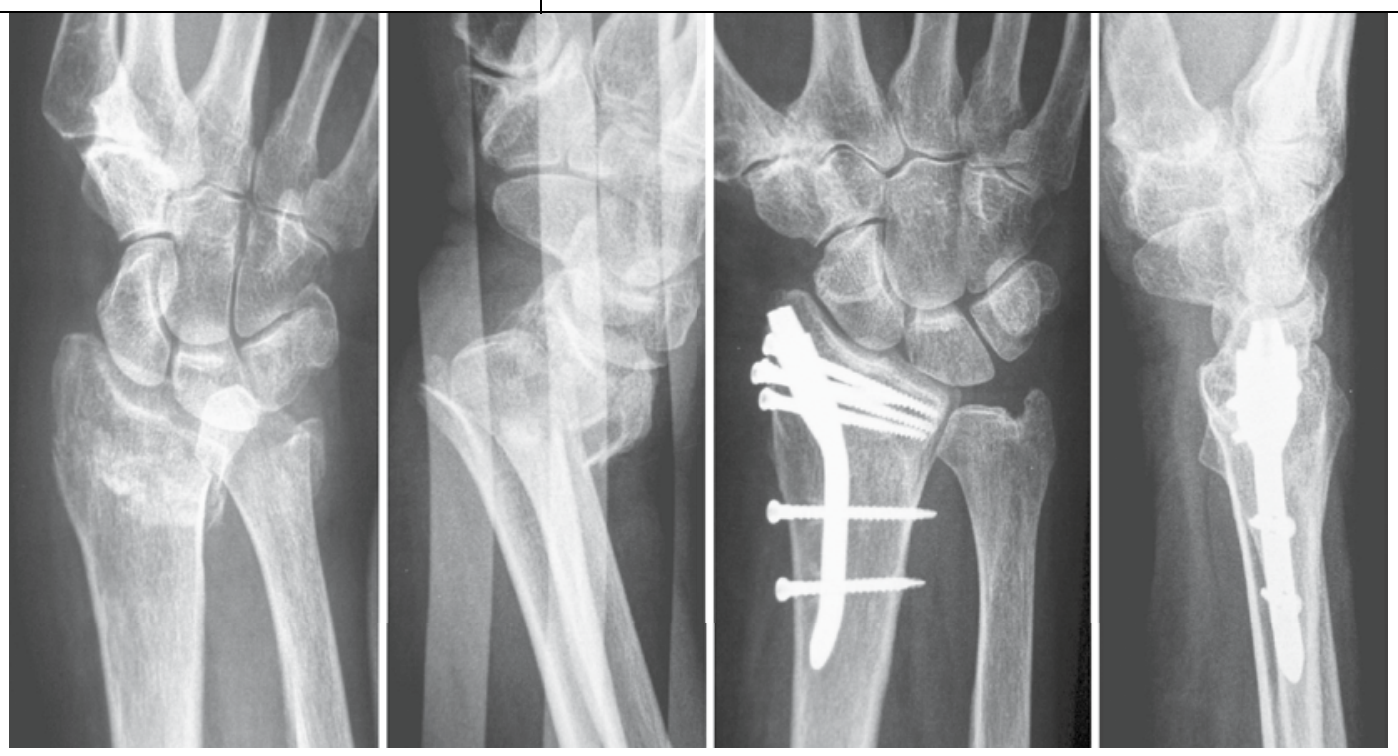

Abb. 1468 -jährige Patientin mit distaler Radiusfraktur AO 23A3.3 präoperativ und 1 Jahr nach intramedullärer Osteosynthese mit Targon $^{\circledast}$ DR (Fa. Aesculap)
- bei einem Wert $<80 \mathrm{mg} \mathrm{HA} / \mathrm{cm}^{3}$ eine Osteoporose.

Die Nachuntersuchung umfasste weiterhin die Erhebung der Funktion beider Handgelenke in sämtlichen Bewegungsebenen mit einem Goniometer, die Messung der Handkraft beidseits mittels Dynamometer sowie die Erhebung des Schmerzniveaus in Ruhe und in Bewegung anhand der VAS (visuelle Analogskala).

\section{Ergebnisse}

122 Patienten (intramedulläre Osteosynthese: $n=66 ; 2,4-m m-L C P: n=56)$ konnten 8 Wochen, 101 Patienten (intramedulläre Osteosynthese: $\mathrm{n}=54 ; 2,4-\mathrm{mm}-\mathrm{LCP}: \mathrm{n}=47$ ) 6 Monate und 61 Patienten (intramedulläre Osteosynthese: $n=37 ; 2,4-\mathrm{mm}$-LCP: $\mathrm{n}=24)$ I Jahr postoperativ nachuntersucht werden. Die intramedulläre Osteosynthese zeichnete sich durch eine kürzere Operationszeit (47 min, p>0,05, Fisher-exaktTest) gegenüber der volaren Plattenosteosynthese ( $55 \mathrm{~min}$ ) aus, war jedoch mit einer längeren Durchleuchtungszeit (intramedulläre Osteosynthese: 2,6 min; 2,4mm-LCP: 1,3 min) verbunden. Die Hospitalisationszeit war in der Gruppe mit intramedullärer Osteosynthese mit 2,5 Tagen gegenüber 4 Tagen bei der Gruppe mit volarer Plattenosteosynthese signifikant kürzer ( $\mathrm{p}>0,05$, Fisher-exakt-Test).

Alle Frakturen heilten, wobei es in keinem der Verfahren zu einem sekundären Repositionsverlust kam. Eine beispielhafte Versorgung ist in $\mathbf{A b b} 1$ gezeigt.
Die Analyse der Röntgenbilder ergab in beiden Gruppen bei jeweils 2 Fällen eine radiale Verkürzung $\geq 2 \mathrm{~mm}$ und eine residuelle Dorsalinklination $\geq 5^{\circ}$. Die intramedulläre Versorgung führte zu einer signifikant schnelleren Restitution der Dorsalextension und Volarflexion und signifikant weniger Schmerz (• Tab. 1). Der mittlere Gartland-and-Werley-Score war 1 Jahr postoperativ nach intramedullärer Osteosynthese $(1,6)$ und volarer Platte $(2,0)$ exzellent.

Mit gleichmäßiger Verteilung zwischen den Gruppen konnten anhand der pQCT-Messung eine osteopenische Spongiosadichte bei $30 \%$ und eine manifeste Osteoporose bei 40\% der Patienten festgestellt werden. Osteoporotisch geschwächter Knochen führte jedoch nicht zu einem Anstieg der Komplikationsquote und hatte keinen Einfluss auf die Handgelenkfunktion.

Bei 2 Patienten nach intramedullärer Osteosynthese und bei 3 Patienten nach volarer Plattenosteosynthese entwickelte sich ein operationspflichtiges Karpaltunnelsyndrom. Obwohl die Implantatentfernung nicht empfohlen wurde, ließen sich 8 von 24 Patienten mit LCP (33\%)und 3 von 37 Patienten mit intramedullärer Osteosynthese (8\%) das Osteosynthesematerial im Rahmen eines ambulanten Eingriffs entfernen.

\section{Diskussion}

Im Rahmen einer prospektiv randomisierten Studie wurde die operative The- rapie von Patienten mit distaler Radiusfraktur mit einem neuen intramedullären Verfahren der volaren winkelstabilen Plattenosteosynthese mit winkelstabiler distaler Schraubenreihe gegenübergestellt. Letztere gehört derzeit zu den Standardverfahren der Radiusfrakturbehandlung $[2,5,8,9,11]$, Erstere dagegen ist ein neuartiges Verfahren, zu welchem nur wenige Berichte vorliegen. Ender-Nägel wurden bei distalen Radiusfrakturen des Erwachsenen in der Vergangenheit gelegentlich eingesetzt, konnten sich jedoch aufgrund der fehlenden Rotationsstabilität nicht durchsetzen [2]. In dieser Studie wurde ein rotationsstabiles Implantat (Targon ${ }^{\circledast}$ DR) getestet, welches durch 4 winkelstabile Schrauben im distalen Fragment einem Hybrid aus Platte und Nagel bzw. einem Marknagel mit Gelenkkomponente entspricht. Das Verfahren erwies sich als stabil, denn es kam in 100\% der Fälle zur Ausheilung der Fraktur ohne sekundären Verlust der Reposition bei sofortigem Beginn der Übungsbehandlung. Tan et al. [13] hingegen sahen in 13\% der Fälle nach Marknagelosteosynthese (Mikronail, Wright Medical Corporation) von Radiusfrakturen ein sekundäres Versagen derselben $[4,13]$.

Patienten, die mit intramedullärer Osteosynthese versorgt wurden, zeigten im Vergleich zur Plattenosteosynthese einen signifikant schnelleren Funktionsaufbau und signifikant weniger Schmerzen in den ersten 8 Wochen nach der operativen Therapie. Dies ist zum einen auf die Operationstechnik zurückzuführen, 
die ein geringeres operatives Trauma darstellt. Der Zugangsweg auf Höhe des Radiusstyloids erfordert eine deutlich geringere Weichteildissektion, da der präformierte Zugang zwischen dem 1. und 2. Strecksehnenfach genutzt wird und der Knochen subkutan liegt. Zum anderen kann man davon ausgehen, dass die intramedulläre Lage des Implantats beim Beugen und Strecken im Handgelenk die Sehnenkrepitation minimiert. Spekulativ bleibt, ob durch den zentralen Kraftträger auch Relativbewegungen in der Fraktur reduziert werden, die bei Plattenosteosynthesen aufgrund der geringeren Steifigkeit und längeren Hebelarme womöglich größer sind.

Gute Ergebnisse der Frakturheilung und postoperativen Beweglichkeit für ein vergleichbares Implantat konnten bereits bei einer limitierten Patientenzahl festgestellt werden [13]. Jedoch zeigte auch die volare winkelstabile Plattenosteosynthese gute funktionelle Ergebnisse, die, abgesehen von den ersten 8 postoperativen Wochen, an die Ergebnisse mit dem Marknagel heranreichen konnten. Rozental u. Blazar [12] berichteten ebenfalls über ein zufrieden stellendes funktionelles Resultat nach volarer Plattenosteosynthese von nach dorsal dislozierten Radiusfrakturen. Interessanterweise wiesen über $6 \%$ ihrer Patienten Synovitisbeschwerden an der Streckseite des Handgelenks auf. Nach Arora et al. [1] können Sehnenkomplikationen bei volaren Plattenosteosynthesen bis zu $16,6 \%$ betragen und somit über die Hälfte der Gesamtkomplikationen darstellen. Ein wesentlicher Grund für Sehnenreizungen an der Streckseite sind Schraubenüberstände und die Form der überstehenden Schraubenspitze, wobei anzunehmen ist, dass bei abgerundeten Spitzen die Affektion der Strecksehnen verringert wird. Ein intramedulläres Implantat mit Platzierung der Schrauben in der Frontalebene kann hingegen das Problem der Synovitis an der Streckseite wirksam reduzieren.

Mit beiden Verfahren ließ sich ein gutes anatomisches Repositionsergebnis erreichen. Problematisch war die korrekte Platzierung der volaren 2,4-mm-Platte auf Höhe der volaren Lippe des Radius, da hierbei die Gefahr einer intraartikulären Schraubenlage besteht [1]. Der Operateur

Trauma Berufskrankh 2008 · 10[Suppl 2]:241-244 DOI 10.1007/s10039-008-1372-2

(C) Springer Medizin Verlag 2008

\section{G. Gradl $\cdot$ M. Wendt $\cdot$ P. Gierer $\cdot$ M. Beck $\cdot$ T. MittImeier Intramedulläre Versorgung der distalen Radiusfraktur}

\section{Zusammenfassung}

Die intramedulläre Versorgung der distalen Radiusfraktur mit einem Hybrid aus Platte und Nagel (Targon ${ }^{\circledast}$ DR) ist ein neues Verfahren. Im Rahmen einer prospektiv randomisierten Studie soll es der volaren winkelstabilen Plattenosteosynthese gegenüber gestellt werden. Bislang wurden 122 Patienten (AO: A2-3, C1-2) eingeschlossen (intramedulläre Osteosynthese: $n=66,2,4-m m-S y n-$ thes-Platte: $\mathrm{n}=56$ ) mit einem mittleren Alter von $63,4 \pm 12,1$ Jahren. Diese wurden klinisch und radiologisch nach 8 Wochen, $6 \mathrm{Mo}$ naten und 1 Jahr nachuntersucht. Alle Frakturen heilten, wobei es bei beiden Verfahren zu jeweils 2 Fällen von radialer Verkürzung um $2 \mathrm{~mm}$ und jeweils $5^{\circ}$ Dorsalinklinationen kam. Die intramedulläre Versorgung führte zu einer signifikant schnelleren Restitution der Dorsalextension und Volarflexion und signifikant weniger Schmerz. Der mittlere Gartland-and-Werley-Score war 1 Jahr postoperativ nach Targon ${ }^{\circledast}$ DR $(1,6)$ und volarer Platte $(2,0)$ exzellent. Die intramedulläre Osteosynthese ist somit ein geeignetes Verfahren zur Behandlung der distalen Radiusfraktur.

\section{Schlüsselwörter}

Distaler Radius · Fraktur · Intramedulläre

Osteosynthese $\cdot$ Plattenosteosynthese $\cdot$ Nagel

\section{Intramedullary osteosynthesis of distal radial fractures}

\section{Abstract}

Intramedullary fixation of distal radial fractures with a plate and nail hybrid (Targon ${ }^{\circledR}$ $\mathrm{DR}$ ) is a new operative technique. A prospective randomized trial is comparing this new technique with volar fixed-angle plating. To date, 122 patients (AO: A2-3, C1-2) have been included (Targon ${ }^{\oplus}$ DR: $\mathrm{n}=66 ; 2.4$ $\mathrm{mm}$ Synthes plate: $\mathrm{n}=56$ ) with a mean age of $63.4 \pm 12.1$ years. Patients were seen for follow-up visits at 8 weeks, 6 months, and 1 year postsurgery. All fractures healed. A residual dorsal tilt of $5^{\circ}$ was noted in five cases each and a loss of radial height by $2 \mathrm{~mm}$ in two cases each. Intramedullary nailing restored the arch of extension and flexion significantly better than volar plating in the early follow-up, and it induced less pain. The Gartland and Werley score averaged 1.6 after Targon ${ }^{\circledR}$ DR and 2.0 after volar plating. Intramedullary nailing is a suitable method for treating distal radial fractures.

\section{Keywords}

Distal radius · Fracture $\cdot$ Intramedullary nailing $\cdot$ Angle plating $\cdot$ Nail 
tendiert somit dazu, die 2,4-mm-Platte leicht distal der „watershed line“ zu positionieren, aber unterhalb der volaren Lippe, was jedoch zu einer signifikanten Reduktion der volaren Kippung führen kann [2]. Die Rekonstruktion der volaren Kippung nach intramedullärer Versorgung zeigte keinen Vorteil gegenüber der volaren Plattenosteosynthese.

\section{Fazit}

Die intramedulläre Osteosynthese stellt ein stabiles Verfahren zur Behandlung der distalen Radiusfraktur dar. Ihr Hauptvorteil liegt in der deutlich geringeren Invasivität, was sich in einer schnellen funktionellen Rekonvaleszenz bemerkbar macht. Der Einsatz bei Frakturen mit mehrfacher Gelenkbeteiligung wurde in diesem Kollektiv nicht untersucht und stellt eine mögliche Limitation der Methode dar.

\section{Korrespondenzadresse}

\section{PD Dr. G. Gradl}

Abteilung für Unfall-

und Wiederherstellungschirurgie, Chirurgische Klinik und Poliklinik, Universität Rostock,

Schillingallee 35, 18055 Rostock georg.gradl@med.uni-rostock.de

Interessenkonflikt. Der korrespondierende Autor weist auf folgende Beziehung/en hin: Referententätigkeit Fa. Aesculap.

\section{Literatur}

1. Arora R, Lutz M, Hennerbichler A et al. (2007) Complications following internal fixation of unstable distal radius fracture with a palmar locking-plate. J Orthop Trauma 21: 316-322

2. Arora R, Lutz M, Zimmermann R et al. (2007) Limits of palmar locking plate osteosynthesis of unstable distal radius fractures. Handchir Mikrochir Plast Chir 39: 34-41

3. Bennett GL, Leeson MC, Smith BS (1989) Intramedullary fixation of unstable distal radius fractures. A method of fixation allowing early motion. Orthop Rev 18: 210-216

4. Brooks KR, Capo JT, Warburton M et al. (2006) Internal fixation of distal radius fractures with novel intramedullary implants. Clin Orthop 445: 42-50

5. Chen NC, Jupiter JB (2007) Management of distal radial fractures. J Bone Joint Surg Am 9: 20512062

6. Gartland JJ, Werley CW (1951) Evaluation of healed Colles' fractures. J Bone Joint Surg Am 33A: 895907

7. Genant H, Block JE, Steiger P et al. (1987) Quantitative computed tomography in assessment of osteoporosis. Semin Nucl Med 17: 316-333
8. Jupiter JB (1991) Current concepts review. Fractures of the distal end of the radius. J Bone Joint Surg Am 73-A: 461-469

9. Jupiter JB (1997) Complex articular fractures of the distal radius: classification and management. J Am Acad Orthop Surg 5: 119-129

10. Lindemann-Sperfeld L, Pilz F, Marintschev I et al. (2003) Fractures of the distal radius. Minimally invasive pin fixation: indications and results. Chirurg 74: 1000-1008

11. Musgrave DS, Idler RS (2005) Volar fixation of dorsally displaced distal radius fractures using the 2.4$\mathrm{mm}$ locking compression plates. J Hand Surg [Am] 30A: 743-749

12. Rozental TD, Blazar PE (2006) Functional outcome and complications after volar plating for dorsally displaced, unstable fractures of the distal radius J Hand Surg [Am] 31: 359-365

13. Tan V, Capo J, Warburton M (2005) Distal radius fracture fixation with an intramedullary nail. Tech Hand Up Extrem Surg 9: 195-201 